\title{
Density functional and Monte Carlo studies of sulfur. II. Equilibrium polymerization of the liquid phase
}

\author{
P. Ballone \\ Institut für Festkörperforschung, Forschungszentrum Jülich, D-52425 Jülich, Germany and Università degli \\ Studi di Messina, Dipartimento di Fisica, Contrada Papardo, I-98166 Messina, Italy \\ R. O. Jones ${ }^{\text {a) }}$ \\ Institut für Festkörperforschung, Forschungszentrum Jülich, D-52425 Jülich, Germany
}

(Received 7 July 2003; accepted 28 July 2003)

\begin{abstract}
The equilibrium polymerization of sulfur is investigated by Monte Carlo simulations. The potential energy model is based on density functional results for the cohesive energy, structural, and vibrational properties as well as reactivity of sulfur rings and chains [Part I, J. Chem. Phys. 118, 9257 (2003)]. Liquid samples of 2048 atoms are simulated at temperatures $450 \leqslant T \leqslant 850 \mathrm{~K}$ and $P=0$ starting from monodisperse $\mathrm{S}_{8}$ molecular compositions. Thermally activated bond breaking processes lead to an equilibrium population of unsaturated atoms that can change the local pattern of covalent bonds and allow the system to approach equilibrium. The concentration of unsaturated atoms and the kinetics of bond interchanges is determined by the energy $\Delta E_{b}$ required to break a covalent bond. Equilibrium with respect to the bond distribution is achieved for $15 \leqslant \Delta E_{b}$ $\leqslant 21 \mathrm{kcal} / \mathrm{mol}$ over a wide temperature range $(T \geqslant 450 \mathrm{~K})$, within which polymerization occurs readily, with entropy from the bond distribution overcompensating the increase in enthalpy. There is a maximum in the polymerized fraction at temperature $T_{\max }$ that depends on $\Delta E_{b}$. This fraction decreases at higher temperature because broken bonds and short chains proliferate and, for $T$ $\leqslant T_{\max }$, because entropy is less important than enthalpy. The molecular size distribution is described well by a Zimm-Schulz function, plus an isolated peak for $\mathrm{S}_{8}$. Large molecules are almost exclusively open chains. Rings tend to have fewer than 24 atoms, and only $S_{8}$ is present in significant concentrations at all $T$. The $T$ dependence of the density and the dependence of polymerization fraction and degree on $\Delta E_{b}$ give estimates of the polymerization temperature $T_{f}$ $=450 \pm 20$ K. (C) 2003 American Institute of Physics. [DOI: 10.1063/1.1611475]
\end{abstract}

\section{INTRODUCTION}

Polymerization is the generic name for a wide variety of reactions involving inorganic, organic, and biological reagents and products. ${ }^{1}$ Monomers or oligomers usually polymerize because bond formation decreases the system enthalpy, but polymerization can occur with increasing enthalpy, if the entropy gain overcompensates the potential energy disadvantage of the polymer phase. In these cases, polymerization takes place above a minimum temperature (floor temperature, $T_{f}$ ), and the system reverts to the monomeric or oligomeric state by cooling below $T_{f}$.

Elemental sulfur is known for the large number of solid allotropes it forms at relatively low temperature and pressure. ${ }^{2-4}$ At the melting point $T_{m}=386 \mathrm{~K},{ }^{5}$ these forms give way to a liquid phase comprising $\mathrm{S}_{8}$ molecules with small concentrations of $\mathrm{S}_{n}$ rings with $6 \leqslant n \leqslant 23 .{ }^{6,7}$ At $T_{\lambda}$ $=432 \mathrm{~K}$, sulfur undergoes a liquid-liquid transition ( $\lambda$ transition) usually interpreted as the ring opening polymerization (ROP) of $\mathrm{S}_{8}$. This transformation, a simple example of entropy driven polymerization, is revealed by a change of color, ${ }^{8}$ anomalies in the specific heat, ${ }^{9,10}$ and volume expansion coefficient ${ }^{11}$ and a dramatic increase of viscosity. ${ }^{12,13}$

\footnotetext{
a) Author to whom correspondence should be addressed. Electronic mail: r.jones@fz-juelich.de
}

Other properties of the system (such as the structure factor) are less affected. ${ }^{14,15}$ The transition is reversible, but with significant hysteresis and a long equilibration time, especially in the cooling branch. The degree of polymerization has been measured recently using Raman spectroscopy at temperatures up to $573 \mathrm{~K} .{ }^{16}$ The kinetics of the transition may be extremely slow also on heating purified $\mathrm{S}_{8}$ samples. Polymerization is enhanced greatly by the presence of even small amounts of $\mathrm{S}_{6}$ and $\mathrm{S}_{7}$ molecules. ${ }^{17,18}$

Thermodynamic properties of sulfur close to the transition have been surveyed, ${ }^{19}$ and early theoretical studies based on thermodynamics, kinetic equations, and mean field schemes ${ }^{20-23}$ achieved a reasonable description of the transition. Renormalization group approaches, accounting for fluctuations and non-mean-field features, have been applied by Wheeler and co-workers. ${ }^{24,25}$ A crucial parameter in the theoretical description of polymerization is the concentration $r$ of activated sites at which the molecular topology can change, and polymerization is predicted to be a second-order phase transition in the limit $r \rightarrow 0$. Nonvanishing values of $r$ result into a rounded transition, corresponding to a steep but continuous shift in the chemical equilibrium between monomers and polymers. The rounding of the transition for $r>0$ has been analyzed in recent mean field studies, ${ }^{26}$ which also emphasized the relationship between polymerization and the 
glass transition. Recent sound velocity and sound attenuation measurements close to the transition ${ }^{27}$ support the conclusion of these mean field studies.

Both mean field and renormalization group approaches focus on the similarities (universal features, scaling relations, etc.) of sulfur polymerization to other systems and transformations, rather than on the features (structure, interatomic forces) that distinguish sulfur from other materials. Here we investigate sulfur polymerization by simulations starting from a quantitative description of bonding and reactivity provided by experiments and by parameter-free density functional (DF) computations.

Experimental studies provide much information on the energetics ${ }^{4,6}$ and structure ${ }^{14}$ of the crystal and liquid phases, as well as detailed investigations of gas phase molecular species. ${ }^{28}$ Detailed information on bonding is also given by computational studies, which include extensive investigations of neutral and charged sulfur molecules, ${ }^{29}$ as well as polymeric chains. ${ }^{30}$ An early analysis ${ }^{31}$ of neutral $S_{n}$ molecules (up to $n=13$ ) by DF methods has been extended recently (to $n=18$ ) and to the analysis of reactivity for diradical sulfur chains. ${ }^{32}$ This study provides the basis for the model used in the present investigation, and the relevant results can be summarized as follows: the $\mathrm{S}-\mathrm{S}$ bonding in sulfur rings is remarkably transferable from one molecule to another, most of the differences and trends observed as a function of ring size being due to the strain induced by the ring topology. The effect of strain is largest in the smallest molecules, while beyond $n \sim 12$ the variation with $n$ of cohesive energy and vibrational properties is a slight even-odd alternation.

Ring opening gives rise to triplet diradical chains. The bonds not immediately adjacent to the unsaturated atoms are remarkably similar to those of rings, while the terminal bonds are shorter and stiffer. Moreover, unsaturated atoms can approach close to saturated atoms belonging to other chains or rings, so that bond interchanges can occur between the incoming radical chain termination and the saturated atoms. This basic step in the polymerization of sulfur involves an energy barrier that does not exceed $5.4 \mathrm{kcal} / \mathrm{mol}$ in the gas phase. These features are incorporated into a semiempirical potential model that is used here for a Monte Carlo simulation of polymerization.

The DF potential energy surface that is the basis for the present model enters explicitly a molecular dynamics simulation of sulfur polymerization ${ }^{33}$ using the method of Car and Parrinello. ${ }^{34}$ This avoids the approximations involved in fitting an empirical potential to the DF data, but the system sizes and simulation times covered by DF based simulations are too limited to provide a quantitative analysis of such a complex transition.

This work is part of a continuing study of equilibrium polymerization. Sulfur displays intriguing analogies to the behavior of bisphenol A polycarbonate (BPA-PC), which also gives rise to rings of different sizes, with the effects of strain apparent in the smallest species. ${ }^{35}$ Moreover, BPA-PC undergoes ring opening polymerization which is an alternative method for preparing high-molecular-weight polycarbonates. The major difference between the systems is that in
BPA-PC the number of bonds is conserved, and a catalyst is required to activate the bond switching dynamics. In the case of sulfur, temperature gives rise to a fluctuating number of broken bonds and unsaturated atoms that initiate polymerization. Furthermore, bending and torsional force constants have been neglected in the study of polycarbonates. Their inclusion here allows a first assessment of their role in the polymerization transition.

\section{INTERATOMIC POTENTIAL}

\section{A. Fit of the interatomic potential}

The potential energy of the system as a function of the interatomic coordinates $\left\{\mathbf{R}_{I}, I=1, N\right\}$ is modeled by a potential comprising bonded $\left(U_{b}\right)$ and nonbonded $\left(U_{n b}\right)$ interactions:

$$
U\left[\mathbf{R}_{1}, \ldots, \mathbf{R}_{N}\right]=U_{b}\left[\mathbf{R}_{1}, \ldots, \mathbf{R}_{N}\right]+U_{n b}\left[\mathbf{R}_{1}, \ldots, \mathbf{R}_{N}\right] .
$$

The bonded interactions include stretching, bending, and torsion contributions:

$$
\begin{aligned}
U_{b}\left[\mathbf{R}_{1}, \ldots, \mathbf{R}_{N}\right]= & \frac{1}{2} K_{S} \sum_{\langle i j\rangle}\left(d_{i j}-d_{i j}^{0}\right)^{2}+\frac{1}{2} k_{B} \sum_{\langle i j k\rangle}(\cos \theta \\
& \left.-\cos \theta_{0}\right)^{2}+K_{\tau} \sum_{\langle i j k l\rangle}[A+B \cos \tau \\
& +C \cos 2 \tau+D \cos 3 \tau+E \cos 4 \tau] .
\end{aligned}
$$

Here $d_{i j}=\left|\mathbf{R}_{i}-\mathbf{R}_{j}\right|$, and $\theta$ and $\tau$ are bending and torsion angles, respectively, identified by three $(\langle i j k\rangle)$ and four $(\langle i j k l\rangle)$ atoms connected by consecutive covalent bonds. Each distance, bending, and torsion angle is counted only once in the sums. The $d^{0}$ and $\theta_{0}$ parameters are the equilibrium distance and bending angle in an ideal unstrained structure. In most sulfur systems, particularly small rings, constraints lead to strained ground-state structures with bond distances and angles significantly different from $d^{0}$ and $\theta_{0}$. Nonbonding interactions are described by a Lennard-Jones (LJ) interaction:

$$
U_{n b}=\sum_{i<j}^{\prime} 4 \epsilon\left[\left(\frac{\sigma}{\left|\mathbf{R}_{i}-\mathbf{R}_{j}\right|}\right)^{12}-\left(\frac{\sigma}{\left|\mathbf{R}_{i}-\mathbf{R}_{j}\right|}\right)^{6}\right],
$$

where the prime indicates that atom pairs connected by covalent bonds are excluded from the sum.

Most atoms in our simulations have covalent bonds to two neighbors, with a small (and temperature dependent) fraction adopting an unsaturated configuration with a single covalent bond. We first describe the choice of the potential parameters for the doubly bonded (regular) atoms. The changes required to describe the interactions involving unsaturated (radical) atoms will be described below.

A preliminary determination of the parameters for the bonded interactions was performed by fitting the equilibrium structure and vibrational frequencies of $\mathrm{S}_{8}$ given by $\mathrm{DF}$ computations. ${ }^{32}$ This ring is the most common entity in the crystalline and liquid forms below the polymer transition. $\mathrm{S}_{8}$ is also the smallest strain-free ring, thus allowing us to de- 
TABLE I. Parameters of the potential energy model. Energies are in $\mathrm{kcal} / \mathrm{mol}$, distances are in $\AA$, and angles are in radians.

\begin{tabular}{ccccccccccc}
\hline \hline$K_{s}$ & $d_{0}$ & $K_{b}$ & $\Theta_{0}$ & $A$ & $B$ & $C$ & $D$ & $E$ & $\sigma$ & $\epsilon$ \\
\hline 210 & 2.109 & 120 & 1.911 & 0 & 1.4824 & 8.0179 & -0.7644 & -4.2000 & 3.36 & 0.44 \\
\hline \hline
\end{tabular}

termine the harmonic contribution for the different energy terms because its vibrational modes are largely separated into stretching, bending, and torsion bands. Anharmonic contributions are not important for stretching and bending, since the high value of the force constants limits the amplitude of fluctuations to narrow intervals around the potential energy minimum. The torsional potential, however, is much softer, and the full range of angles might be explored by the system in the temperature range of interest (up to about $1000 \mathrm{~K}$ ). Moreover, the anharmonic part of this contribution might be important for properties such as thermal expansion, melting, and the $\lambda$ transition. For these reasons, the initial model of intramolecular interactions has been refined by fitting equilibrium geometries and energies (relative to the $\mathrm{S}_{8}$ groundstate energy) obtained by DF computations for $\mathrm{S}_{n}$ rings with $4 \leqslant n \leqslant 18$. The parameters of the LJ potential are determined by fitting the experimental ground-state volume and cohesive energy of $\alpha$-sulfur. All parameters are listed in Table I.

The polymerization of sulfur takes place in two steps:

(1) Initiation:

cyclo- $\mathrm{S}_{8} \rightarrow$ chain- $\mathrm{S}_{8}$.

(2) Propagation:

chain- $\mathrm{S}_{8}+n \mathrm{~S}_{8} \rightarrow \mathrm{S}_{\text {poly }}^{.}$.

The reaction enthalpy for initiation $\left(\Delta H_{I}\right)$ has been estimated by electron spin resonance (ESR) measurements above $823 \mathrm{~K}$ to be $36.4 \mathrm{kcal} / \mathrm{mol}$, in good agreement with static magnetic susceptibility measurements up to $873 \mathrm{~K}$ $(34.9 \mathrm{kcal} / \mathrm{mol})$. The enthalpies of the initiation and propagation $\left(\Delta H_{P}\right)$ step have been estimated by Tobolsky and Eisenberg $^{21}$ to be 32.8 and $3.17 \mathrm{kcal} / \mathrm{mol}$, respectively. A more recent estimate of the propagation enthalpy yields $\Delta H_{P}=4.5 \mathrm{kcal} / \mathrm{mol} .{ }^{18}$ To reproduce these effects we introduce bond breaking and formation, which are assumed to be discontinuous processes; each pair of atoms is either bonded or not bonded at a given time, and their bonding state (represented by an independent binary variable) is not determined uniquely by the interatomic distance. In this respect, the model differs significantly from one where atoms move on a continuous potential energy surface and the bonding pattern is defined by interatomic distances alone. ${ }^{36}$

The energy $\Delta E_{b}$ is required to break a bond and is released on bond formation. A simple estimate shows that the fraction of broken bonds at temperatures below $\sim 1000 \mathrm{~K}$ is very small if $\Delta E_{b}$ is identified with the experimental reaction enthalpy $\Delta H_{I}$ of the initiation step for polymerization, and either a very large system or very long simulations would be required to observe one or more events that could trigger polymerization. We view $\Delta E_{b}$ as a free parameter in a sequence of simulations $\left(\Delta E_{b}=15,18,20\right.$, and $\left.21 \mathrm{kcal} / \mathrm{mol}\right)$ from which the limit $\Delta E_{b} \rightarrow \Delta H_{I}$ can be investigated. The concentration of unsaturated atoms resulting from bond breaking processes plays the role of the parameter $r$ in Ref. 26 , defined as the concentration of initiator particles. As such, it determines the sharpness of the transition and affects structural properties of the polymer phase, most notably the average length of open chains.

Broken bonds open the way to propagation, which takes place via a bond switching mechanism involving one unsaturated atom (see Fig. 1), and the corresponding energy barrier depends on the interaction of the radical atom with all others. If this interaction is the same as for regular atoms, bond switching would not be observed during runs of practicable length. However, DF computations ${ }^{32}$ show that radical atoms can approach regular atoms much closer than a pair of regular atom, and the energy barrier is relatively low. To reproduce these features, the LJ energy parameter $\epsilon$ for saturated atoms has been multiplied by 1.8 , while the $\mathrm{LJ}$ diameter $\sigma$ has been halved. This choice greatly extends the temperature range for which equilibrium with respect to bond interchanges is achieved. By computing the average rate of bond interchanges as a function of we have shown that the corresponding free energy barrier for the propagation step is consistent with the DF estimate. ${ }^{32}$

\section{B. Test of the interatomic potential}

The potential energy function for sulfur is a simple flexible bond model and less detailed than some used previously. ${ }^{37}$ The DF data set and the accumulated experience of fitting molecular potential functional forms would allow an improved description of sulfur structure and dynamical properties. However, we need to perform millions of Monte Carlo (MC) moves per atom on systems with more than $\sim 1000$ atoms, so that our model describes satisfactorily

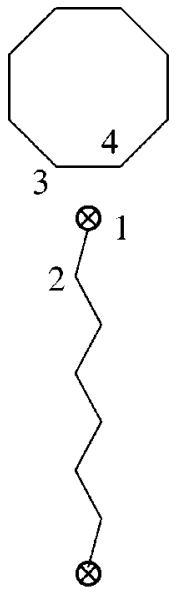

( a )

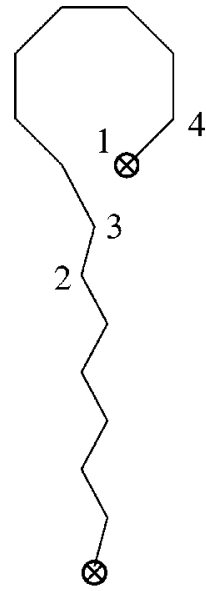

( b )
FIG. 1. Schematic view of the bond interchange mechanism. The large circles with a cross represent unsaturated atoms. 
TABLE II. Harmonic vibrational frequencies $\left(\right.$ in $\mathrm{cm}^{-1}$ ) of $\mathrm{S}_{8}$ computed by DF (Ref. 32) and the model potential.

\begin{tabular}{rrr}
\hline \hline Mode & DF & Model pot. \\
\hline 1 & 73.13 & 73.88 \\
2 & 73.73 & 73.88 \\
3 & 135.37 & 138.30 \\
4 & 136.03 & 138.30 \\
5 & 184.37 & 186.70 \\
6 & 185.47 & 191.20 \\
7 & 207.80 & 191.20 \\
8 & 231.17 & 212.92 \\
9 & 239.26 & 212.92 \\
10 & 242.38 & 245.55 \\
11 & 328.56 & 377.59 \\
12 & 372.04 & 377.59 \\
13 & 373.37 & 388.11 \\
14 & 436.14 & 409.55 \\
15 & 436.35 & 409.55 \\
16 & 442.39 & 441.29 \\
17 & 443.88 & 441.29 \\
18 & 454.41 & 455.45 \\
\hline \hline
\end{tabular}

a range of properties of sulfur. The harmonic vibrational frequencies of $\mathrm{S}_{8}$ given by the model potential are compared with the DF values ${ }^{32}$ in Table II. The correspondence of eigenvectors is satisfactory for stretching and bending modes, somewhat less so for the lowest-frequency modes. The cohesive energy per atom for a sequence of $S_{n}$ rings is shown in Fig. 2. The main features of the DF calculations ${ }^{32}$ are again reproduced by the potential.

The equation of state of $S_{8}$ condensed phases as a function of temperature has been computed by MC in the NPT ensemble (at $P=0$ ) for a sample of 256 molecules, initially in the $\alpha$-S structure (space group $D_{2 h}^{24}, F d d d$ ). This structure is stable in our simulations up to $\sim 400 \mathrm{~K}$, where fluctuations in the simulation box shape make it difficult to identify the crystal phase. The potential energy as a function of temperature is shown in Fig. 3. The bonding is fixed during these simulations, so that the system comprises $\mathrm{S}_{8}$ molecules alone. The linear thermal expansion coefficient in the crystal

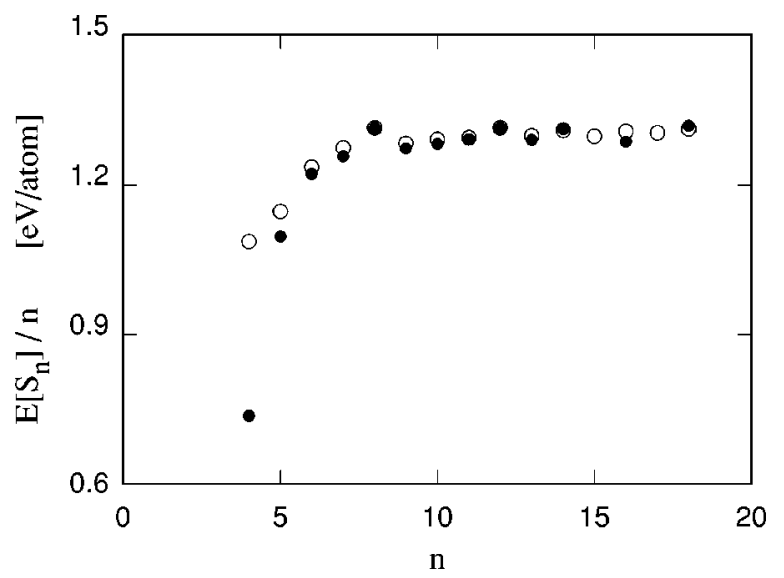

FIG. 2. Cohesive energy per atom of sulfur rings as a function of size. Solid dots: results of the empirical model discussed in Sec. II. Open circles: DF results from Ref. 32 .

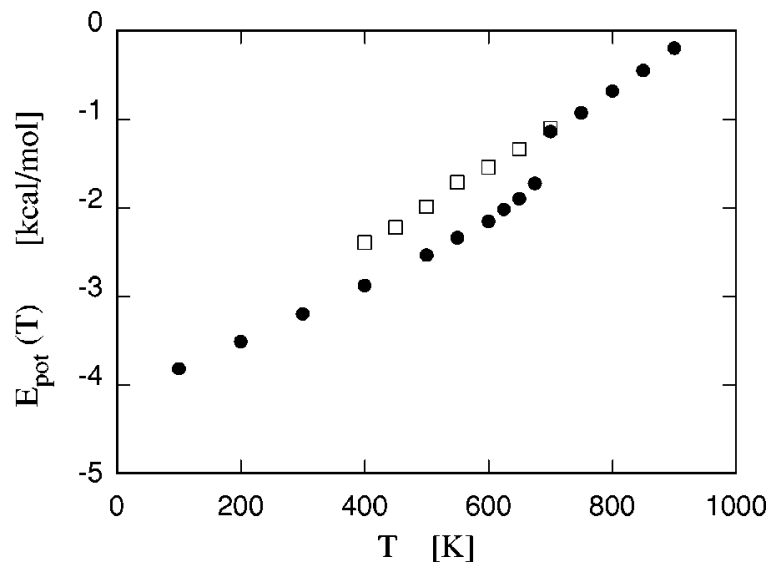

FIG. 3. Average potential energy per atom of condensed $S_{8}$ molecular systems as a function of $T$ at $P=0$. Solid dots: samples heated from an $\mathrm{S}_{8}$ crystal at $T=0 \mathrm{~K}$. Open squares: samples obtained by quenching liquid systems from $T=750 \mathrm{~K}$. Each point is the average over $10^{6} \mathrm{MC} /$ steps per atom, following an equilibration run of equal length. Bond breaking and bond interchange are not allowed during these runs. The error bars are comparable to the size of the symbols.

phase $\left(\alpha_{T}=5.7 \times 10^{-5} \mathrm{~K}^{-1}\right)$ is in satisfactory agreement with experimental data $\left(\alpha_{T}=6.4 \times 10^{-5} \mathrm{~K}^{-1}\right){ }^{38}$

The discontinuities in $U(T)$ and $V(T)$ at $T_{L} \sim 650 \mathrm{~K}$ identify the temperature at which the simulated samples become liquid, as confirmed by the analysis of the atomic mean square displacement as a function of the length of the MC simulation. Hysteresis means that $T_{L}$ cannot be identified with the melting temperature $T_{M}$ of the model potential, which must be computed via the comparison of free energies for the crystal and the liquid phases. Experience with molecular systems, however, suggests that $T_{L} \gg T_{M}$. The metastability of the liquid phase below $T_{L}$ allows us to prepare fluid samples for $T \geqslant 450 \mathrm{~K}$ by quenching liquid samples from above $T_{L}$. No quenched samples showed any tendency to crystallize, and a liquidlike behavior of the atomic meansquare displacement was apparent down to the lowest temperature $(T=450 \mathrm{~K})$ reached by quenching.

The radial distribution function in the liquid phase is in fair agreement with experimental results. All qualitative features (including the trends across the crystal to liquid transition) are reproduced, but the radii of the first few peaks of the radial distribution function are overestimated by about 4\% [experimental data are from Ref. 14(b)]. Half of the discrepancy is probably due to the slight overestimation of bond lengths by DF computations, but this discrepancy should not affect the results of the polymerization reaction, and we have not modified the potential obtained by fitting the DF data.

\section{MONTE CARLO SIMULATIONS OF POLYMERIZATION}

\section{A. Method}

The Monte Carlo method is used for simulating the polymerization reaction, because the discontinuous bond breaking and forming and bond switching processes make the development of an efficient and reliable molecular dynamics implementation difficult. The MC procedure includes different types of moves: Single atom moves are attempted using 
standard Metropolis MC, and whole molecule moves are attempted as well. The list of atoms forming the molecule is determined before each move, because the bonding configuration changes during the simulation. The step size is the same for all molecules, but moves involving molecules larger than 200 atoms are rejected, since the acceptance probabilities would be very small. All simulations are performed in the NPT ensemble at $P=0 .{ }^{39}$ In the case of liquid and polymeric samples, the Andersen algorithm (uniform scaling of the unit cell, at constant angles among the primitive vectors) is used. ${ }^{40}$ The step size for all these moves is such that the acceptance probability is close to 0.5 .

Bond breaking and formation are attempted with the same frequency as the single atom moves, but their acceptance ratio is orders of magnitude smaller. Care is taken to enforce microscopic reversibility. For each bond breaking attempt, an atom is selected at random, and the move is rejected if this atom has only one covalent bond (i.e., every atom is at least singly coordinated). Otherwise, another atom is selected randomly within a sphere of radius $R=3 \AA$ centered on the first. Bond breaking is attempted if this second atom has a covalent bond to the first, and accepted or rejected with probability given by the Boltzmann factor for the energy change (including $\Delta E_{b}$ ). An equivalent strategy is used for bond formation: An atom is selected at random and, if twofold coordinated, the move is rejected. Otherwise, a second atom is selected at random within a sphere of radius $R$ centered on the first. If this second atom is also engaged in only one bond, a covalent bond is formed between the first and second atom. The attempted change in the bonding pattern is followed by an acceptance-rejection decision based on the Metropolis rules.

In principle, bond breaking and formation are sufficient to establish equilibrium with respect to the bonding configuration, but the kinetics are exceedingly slow for realistic choices of $\Delta E_{b}$. Bond switching processes also lead to equilibrium, with a significantly lower energy barrier. Care must also be taken here to ensure microscopic reversibility. Here the bond switching process is attempted by selecting an atom at random. If it is unsaturated, a second atom is selected at random within a distance $R$ from the first, and the move is rejected if the second atom is also unsaturated. Otherwise, a bond interchange is attempted following the pattern shown in Fig. 1. Acceptance or rejection is then decided according to the standard $\mathrm{MC}$ rules. This process must overcome the barrier $\Delta E_{c}$, which is considerably lower than $\Delta E_{b}$, but a significant reaction rate requires the presence in the system of a sizable concentration of unsaturated atoms, which depends in turn on $\Delta E_{b}$.

Values of $\Delta E_{b}$ lower than $15 \mathrm{kcal} / \mathrm{mol}$ lead to an unrealistically large number of broken bonds for $T \geqslant 450 \mathrm{~K}$ and a significant increase in the system volume. If, however, the number of broken bonds in the simulation cell decreases below the critical number of 1 for $\Delta E_{b}>21 \mathrm{kcal} / \mathrm{mol}$ and $T$ $\sim 500 \mathrm{~K}$, the system does not reach equilibrium. For accessible values $\left(15 \leqslant \Delta E_{b} \leqslant 21 \mathrm{kcal} / \mathrm{mol}\right)$ the results of the simulation depend significantly on $\Delta E_{b}$, and we perform a sequence of simulations spanning the interval $450 \leqslant T$ $\leqslant 800 \mathrm{~K}$ for each of the values for $\Delta E_{b}=15,18,20$ and 21 $\mathrm{kcal} / \mathrm{mol}$. This provides a basis for the extrapolation of the results to the physical value $\Delta E_{b} \sim 35 \mathrm{kcal} / \mathrm{mol}$.

Convergence with respect to the bond configuration has been monitored by computing the probability distribution as a function of cluster size. Full equilibration requires from $10^{6}$ single-particle moves per atom at the highest temperatures to $10 \times 10^{6}$ moves per atom at the lowest. The averages reported below have been computed over runs of length equal to that of the equilibration stage under the same conditions. We have noted that the number of attempts of bond interchange and bond breaking and formation attempts is equal to the total number of single-atom moves; i.e., for either equilibration or production runs it ranges from 2048 $\times 10^{6}$ at the highest temperatures to $2048 \times 10^{7}$ at the lowest temperatures.

\section{B. Results}

Every simulation began from a sample comprising $\mathrm{S}_{8}$ rings, with all atoms forming two covalent bonds. Initial configurations were taken from the constant-pressure simulations for $\mathrm{S}_{8}$ rings discussed in Sec. II. Configurations were selected from the heating branch of Fig. 3 for $T \geqslant 700 \mathrm{~K}$ and from the cooling branch for $T<700 \mathrm{~K}$. This means that all samples were in a (stable or metastable) fluid phase.

The relatively high values of $\Delta E_{b}$ limit the number of broken bonds and the number of atoms able to interchange bonding. Together with the non-negligible barrier for bond interchanges, this implies that the biggest problem in these simulations is to reach equilibrium with respect to the average number of broken bonds and the bond distribution. Several independent diagnostic quantities show that equilibrium was achieved for $T \geqslant 450 \mathrm{~K}$ for $\Delta E_{b}=15 \mathrm{kcal} / \mathrm{mol}, T$ $\geqslant 500 \mathrm{~K}$ for $\Delta E_{b}=18 \mathrm{kcal} / \mathrm{mol}, T \geqslant 550 \mathrm{~K}$ for $\Delta E_{b}=20$, and $T \geqslant 600 \mathrm{~K}$ for $\Delta E_{b}=21 \mathrm{kcal} / \mathrm{mol}$. Results for partially equilibrated samples ( $T=450 \mathrm{~K}, \Delta E_{b}=18$ ) will be discussed in connection with the $T$ dependence of the polymerization degree.

In all cases, a long sequence of $\mathrm{MC}$ steps is required before the first broken bond appears within the system. With one or two exceptions (in more than 20 cases) the first pair of unsaturated atoms does not recombine before taking part in bond interchanges, thus beginning the propagation stage of polymerization. We also performed long simulations for crystal-like samples at $T=600 \mathrm{~K}$ and $T=650 \mathrm{~K}$. Bond breaking was also observed in these cases, but the unsaturated atom pairs recombined before exchanging bonds, indicating that a fluidlike environment is a (kinetic or thermodynamic) prerequisite for polymerization.

Bond interchanges occur preferentially at atomic configurations in which one unsaturated chain head approaches two saturated atoms belonging to a ring (see Fig. 4) or another chain. The statistical probability of these configurations is determined by the van der Waals parameters for the cross (i.e., unsaturated-saturated) interactions, which determine the average number of successful bond interchanges. The role of the threefold-coordinated unsaturated atoms at the center of these active sites was noted in earlier work, ${ }^{36}$ where configurations similar to those of Fig. 4 were referred to as tadpoles. The MC kinetics of the polymer growth can be 


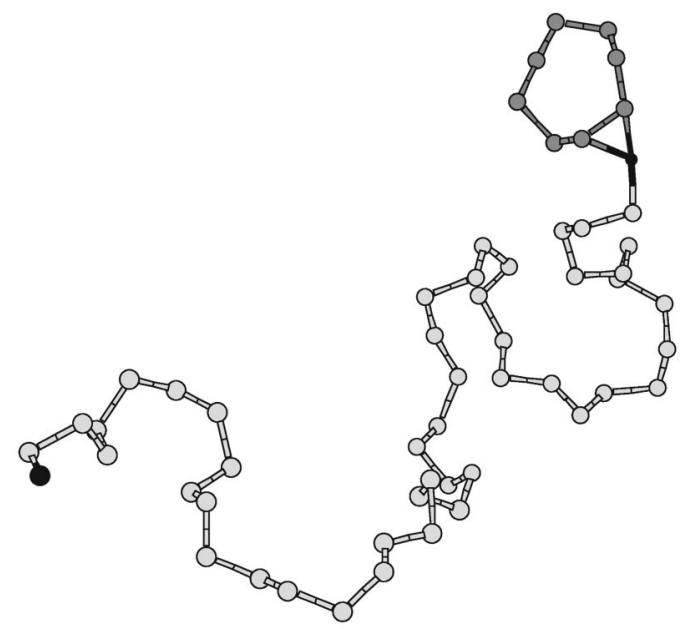

FIG. 4. Snapshot of an activated complex (tadpole) leading to bond interchanges. The complex consists of an $\mathrm{S}_{8}$ ring (dark gray) and an open chain (light gray). Unsaturated atoms are black.

monitored by plotting the number $N_{L}$ of atoms belonging to molecules (chains or rings) with more than $L$ atoms. An example is $N_{24}$, shown in Fig. 5(a) for $T=750 \mathrm{~K}$ and $\Delta E_{b}$ $=21 \mathrm{kcal} / \mathrm{mol}$.

The growth of the polymer phase is accompanied by an increase in the average potential energy of the system [see

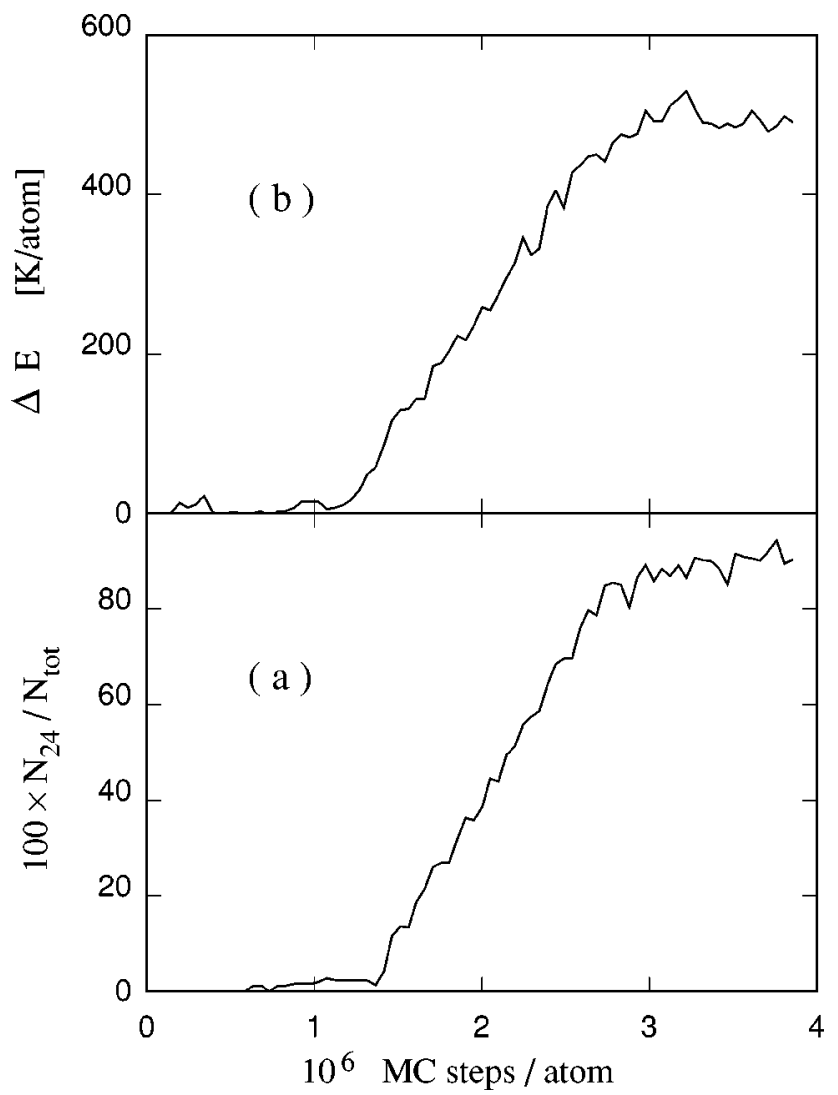

FIG. 5. Evolution of properties as a function of the number of MC steps during polymerization at $T=750 \mathrm{~K}, \Delta E_{b}=21 \mathrm{kcal} / \mathrm{mol}$. (a) Percentage of atoms belonging to molecules with more than 24 atoms. (b) Average potential energy per atom. The zero of the energy is the average potential energy of the molecular $\mathrm{S}_{8}$ phase at the same temperature.

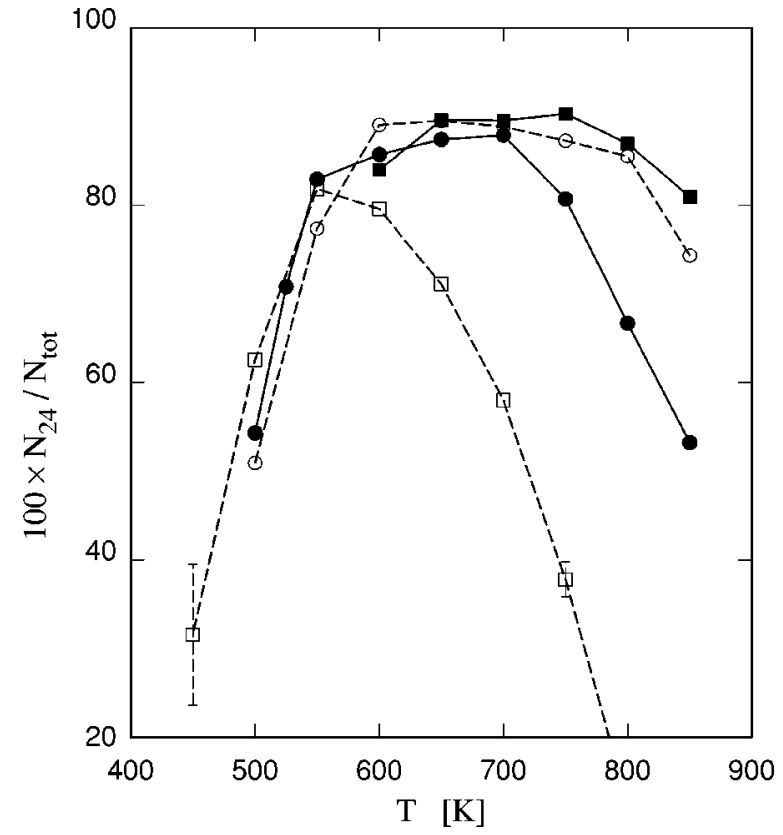

FIG. 6. Average value of $N_{24}$ as a function of $T$. Open squares: $\Delta E_{b}$ $=15 \mathrm{kcal} / \mathrm{mol}$. Solid dots: $\Delta E_{b}=18 \mathrm{kcal} / \mathrm{mol}$. Open circles: $\Delta E_{b}$ $=20 \mathrm{kcal} / \mathrm{mol}$. Solid squares: $\Delta E_{b}=21 \mathrm{kcal} / \mathrm{mol}$. Representative error bars are shown. Lines are a guide to the eye.

Fig. 5(b)]. This is not a transient effect due to the reaction, but an equilibrium property of the polymer, which is stable despite its energy disadvantage with respect to the molecular phase. The energy required to break bonds accounts for $25 \%$ of the total energy increase shown in Fig. 5(b) for $T=750 \mathrm{~K}$ and $\Delta E_{b}=21 \mathrm{kcal} / \mathrm{mol}$. In real experiments this contribution is very small, because $\Delta E_{b}$ is higher than the values used in the simulations, thus depressing exponentially the number of broken bonds and of the energy associated to them. Subtracting the broken bond energies from the enthalpy increase measured in going from the molecular to the polymer phase, we obtain $\Delta H_{P}=322,346,370,403,428$, and $448 \mathrm{~K} /$ atom for $T=600,650,700,750,800$, and $850 \mathrm{~K}$, respectively. A linear extrapolation to $T=450 \mathrm{~K}$ gives $\Delta H_{P}(T=450 \mathrm{~K})=242 \mathrm{~K} /$ atom, equal to $3.85 \mathrm{kcal} / \mathrm{mol} \mathrm{S} \mathrm{S}_{8}$, in fair agreement with the estimate $\left(\Delta H_{P}=4.5 \mathrm{kcal} / \mathrm{mol}\right)$ based on experimental data. ${ }^{18}$

The potential energy increase makes it easier to break bonds, since the number of unsaturated atoms grows steadily during polymerization, at a rate far exceeding that observed during polymer nucleation. The peculiar relation between polymerization and bond breaking is discussed below. Here we emphasize that the rapid increase of unsaturated atoms upon polymerization is instrumental in reaching equilibrium in most of the simulations.

A polymeric molecule is defined here as a chain or ring with more than 24 atoms, so that the equilibrium value of $N_{24}$ is a measure of the polymerized fraction. ${ }^{41}$ This is shown in Fig. 6 as a function of $T$ for four values of $\Delta E_{b}$. There is a maximum in the polymer fraction at a temperature $T_{\max }$ that grows with increasing $\Delta E_{b}$. Further temperature increase leads to a significant decrease in $N_{24}$, because the exponential increase in the number of unsaturated atoms pro- 


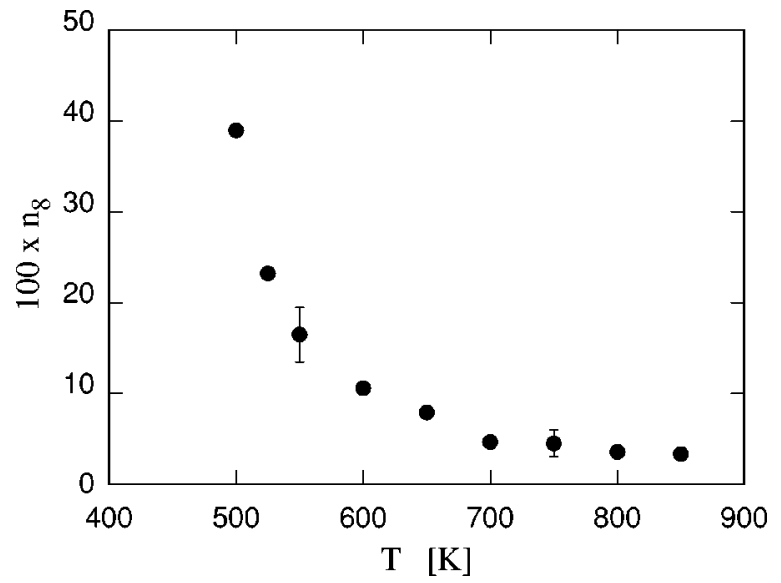

FIG. 7. Fraction of the system mass (in percent) represented by $S_{8}$ rings as a function of $T$ for $\Delta E_{b}=18 \mathrm{kcal} / \mathrm{mol}$. Representative error bars are shown.

duces many shorter segments. The decrease of the polymerization degree at $T<T_{\max }$ is apparently due to the progressive stabilization of $\mathrm{S}_{8}$ rings, whose potential energy advantage with respect to the polymer phase becomes more important at low $T$ (see Fig. 7). Other small rings contribute little to the total system mass at all $T$. A similar relative destabilization of the polymer phase at low $T$ was observed with the polycarbonate model, ${ }^{42,43}$ but the two models behave differently at high $T$. In the latter, the number of bonds and active particles is conserved, and the thermal destabilization of large molecules is not observed.

Available experimental data do not provide evidence of a maximum in the polymerized fraction, ${ }^{16,27}$ probably because the temperature ranges (up to 560 and $470 \mathrm{~K}$, respectively) do not extend to sufficiently high $T$. However, the mechanisms determining the shape of $N_{24}(T)$ (or of any equivalent measure of the polymerized fraction) are so general that we expect a maximum will be found when experiments are carried out at temperatures comparable to those of the simulations.

The variation of the average length of polymeric molecules as a function of $T$ and $\Delta E_{b}$ reflects the number of broken bonds and the polymer fraction $N_{24}$ (see Fig. 8). We note below that molecules with more than 24 atoms are almost all chains, whose number at any time is equal to the number of broken bonds (or half of the number of unsaturated atoms). Moreover, the number of chains with fewer than 24 atoms is very small in well-polymerized samples (i.e., where $N_{24}$ is higher than 50\%). The average value of $N_{24}$ is then approximately equal to the average length of polymeric molecules times the average number of broken bonds. The average length $\langle L\rangle$ will then decrease faster than the polymer fraction for $T \geqslant T_{\max }$, reflecting the decrease of $N_{24}$ and the increase of the average number of chains with increasing $T$. $\langle L\rangle$ will peak at a temperature $T_{\max }^{\langle L\rangle}$ somewhat lower than $T_{\max }$. On cooling below $T_{\max }^{\langle L\rangle},\langle L\rangle$ will decrease more slowly than $N_{24}$, since the decrease in the polymer fraction with decreasing $T$ is partly compensated by the decrease in the number of unsaturated atoms (and chains).

The difference between $T_{\max }^{\langle L\rangle}$ and the estimated polymerization temperature $\left(T_{\max }^{\langle L\rangle}-T_{f} \sim 50 \mathrm{~K}\right.$, using the $T_{f}=450 \mathrm{~K}$

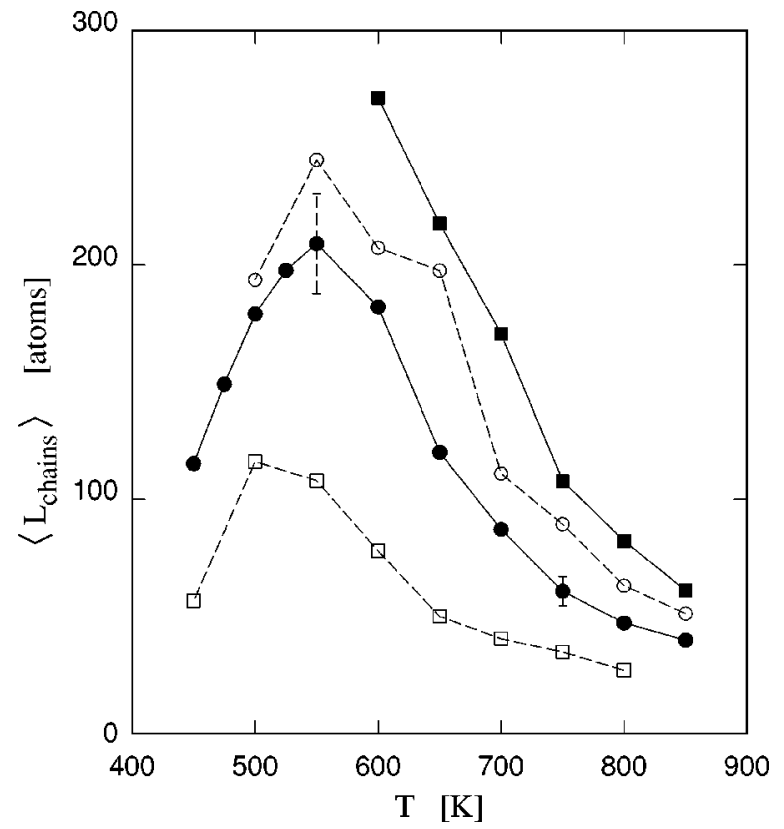

FIG. 8. Average length of polymeric molecules as a function of $T$. Open squares: $\Delta E_{b}=15 \mathrm{kcal} / \mathrm{mol}$. Solid dots: $\Delta E_{b}=18 \mathrm{kcal} / \mathrm{mol}$. Open circles: $\Delta E_{b}=20 \mathrm{kcal} / \mathrm{mol}$. Solid squares: $\Delta E_{b}=21 \mathrm{kcal} / \mathrm{mol}$. Representative error bars are shown. Lines are a guide to the eye.

estimate given below) is somewhat higher than the range $(\sim 10 \mathrm{~K})$ predicted by previous theoretical studies ${ }^{21}$ and by experimental data. ${ }^{12}$ The difference is probably due to the rounding of the transition in our simulations, since lower values of $\Delta E_{b}$ enhance the concentration of unsaturated atoms. The near saturation of $N_{24}$ at $\sim 90 \%$ for $T \sim T_{\max }$ and the exponential decrease of the number of chains with increasing $\Delta E_{b}$ (at constant $T$ ) allow us to predict that at the physical value $\Delta E_{b} \sim 32 \mathrm{kcal} / \mathrm{mol}$ the average length of chains at or below $T_{\max }$ will exceed a few thousand atoms.

The equilibrium size distribution of the sulfur molecules (chains or rings) is characterized by the function $p(L)$, defined as

$$
p(L)=\frac{n(L)}{\sum_{L=1}^{\infty} n(L)},
$$

where $n(L)$ is the average number of molecules whose size is $L$. The function $p(L)$ has been used extensively in theoretical and computational studies and emphasizes the low- $L$ portion of the size distribution. In well-polymerized samples, however, most of the system mass is accounted for by a few large molecules, whose role is underrepresented by the vanishingly small tail of $p(L)$. We therefore show the function $P(L)$ (the mass fraction distribution):

$$
P(L)=100 \frac{L}{\langle L\rangle} p(L) .
$$

A typical result for a polymerized sample is shown in Fig. 9.

A sharp peak is apparent at $L=8$ for all $T$ in both $p(L)$ and $P(L)$. For larger sizes, $p(L)$ is well reproduced by a Zimm-Schulz distribution:

$$
p(L) \propto L^{\gamma-1} \exp [-\gamma L /\langle L\rangle],
$$




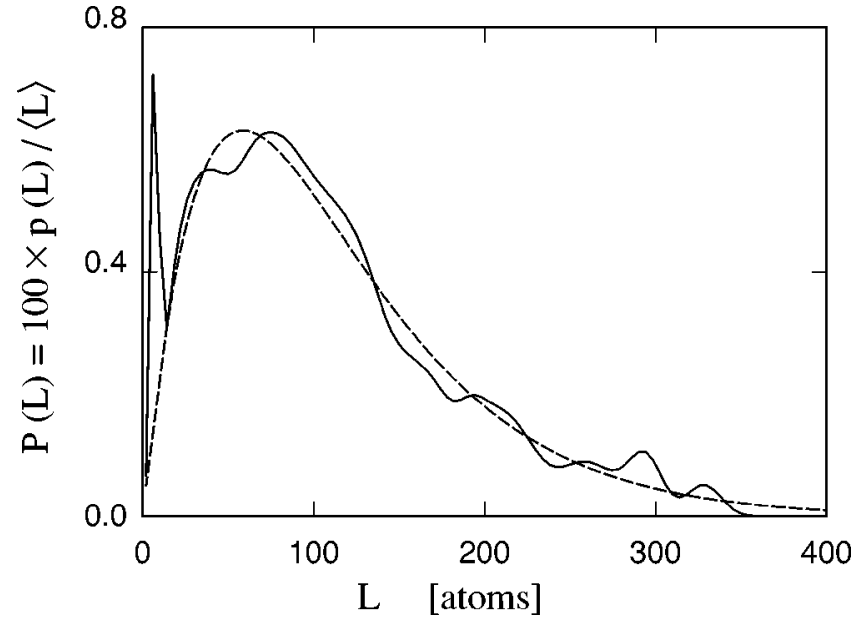

FIG. 9. Mass fraction distribution function $P(L)$ (see text) for $T=800 \mathrm{~K}$ and $\Delta E_{b}=21 \mathrm{kcal} / \mathrm{mol}$. Solid curve: simulation results. Dashed curve: interpolation by a Zimm-Schulz distribution excluding the peak at $L=8$ (see text).

with an exponent $\gamma=1.1$ that is almost independent of $T$ and $\Delta E_{b}$ (see Fig. 9). The simpler function $p(L) \propto \exp [-L /\langle L\rangle]$ provides a fit whose $\chi^{2}$ is almost as good. The weak dependence of $\gamma$ on the simulation parameters implies that the dependence of $p(L)(L>8)$ on $T$ and $\Delta E_{b}$ (see Figs. 10 and 11) follows simply from the above considerations for the average length $\langle L\rangle$.

The peak of $p(L)$ at $L=8$ (see Fig. 9) is not an artifact of incomplete equilibration of the original population of $\mathrm{S}_{8}$ molecules, as we have shown by cycling samples between different temperatures and observing an evolution in the height of the peak. The reversibility of the $S_{8}$ polymerization is shown by the recovery of the $S_{8}$ population upon quenching a well-equilibrated polymerized sample from $T=750 \mathrm{~K}$ to $T=550 \mathrm{~K}$ (Fig. 12). The final state is still polymeric (the polymer fraction measured by $N_{24}$ decreases only slightly from $T=750 \mathrm{~K}$ to $T=550 \mathrm{~K}$ ), but $\mathrm{S}_{8}$ accounts for more than $20 \%$ of the total mass. Because of the difficulty in reaching equilibrium at significantly lower temperatures, the large in-

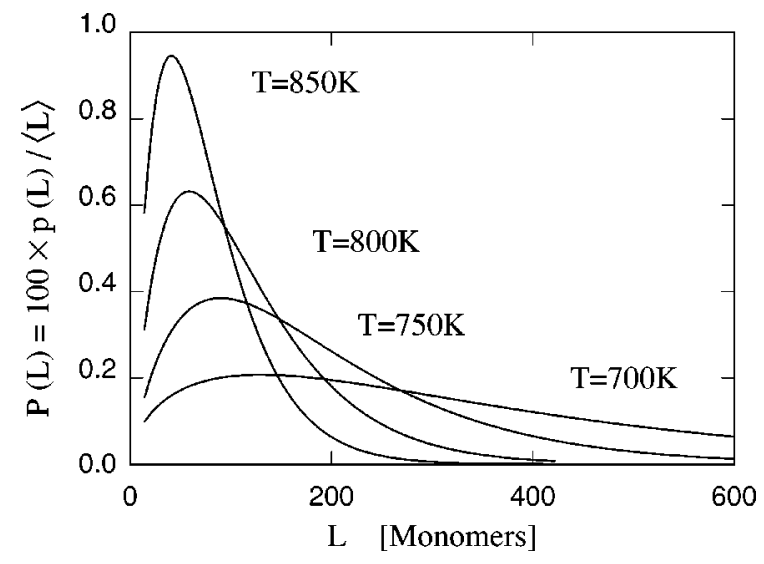

FIG. 10. Mass fraction distribution $P(L)$ (see text) at different $T$ for samples with $\Delta E_{b}=21 \mathrm{kcal} / \mathrm{mol}$. Noise is reduced by showing the ZimmSchulz interpolation of $P(L)$ for $L>8$.

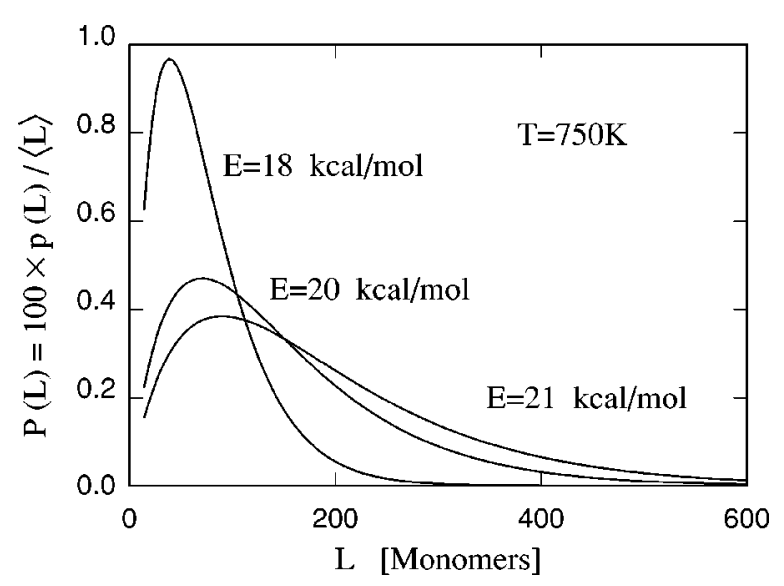

FIG. 11. Mass fraction distribution $P(L)$ (see text) at $T=750 \mathrm{~K}$ for different values of $\Delta E_{b}$. Noise is reduced by showing the Zimm-Schulz interpolation of $P(L)$ for $L>8$.

crease of the $S_{8}$ fraction with decreasing temperature is the strongest indication that the polymer transition displayed by the model is reversible.

The simulation data for $p(L)$ are consistent with the predictions of Ref. 26 (a) [see Eq. (26) in particular] based on a mean field approach and representing the size probability distribution as the superposition of an isolated peak for the monomer (i.e., $\mathrm{S}_{8}$ in the case of sulfur) plus a decaying exponential function for larger sizes.

The $S_{8}$ population is significant at all temperatures. However, the mass fraction of rings is very low in all polymerized samples, and molecular loops with more than 24 atoms are exceptional and statistically irrelevant. ${ }^{44}$ With decreasing $T$, the relative fraction of rings other than $\mathrm{S}_{8}$ increases from $3.0 \%$ at $T=750 \mathrm{~K}$ to $4.6 \%$ at $T=550 \mathrm{~K}$. These results are similar to those of Refs. 42 and 43, with intriguing differences. The mass fraction of rings in sulfur is much lower than in the polycarbonate model if $S_{8}$ is excluded, but

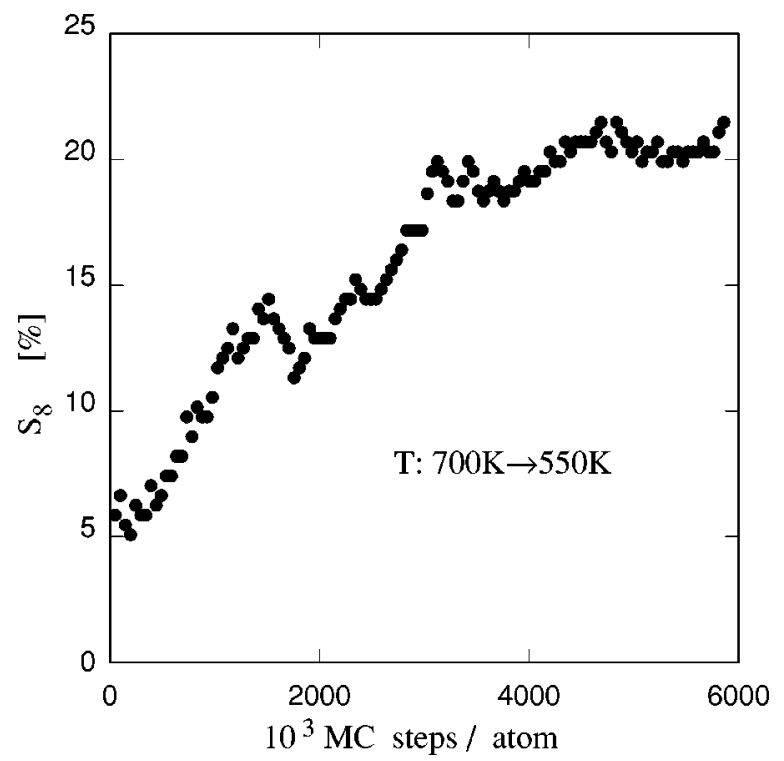

FIG. 12. Fraction of the total mass represented by $S_{8}$ rings as a function of MC steps per atom upon quenching a polymerized system from $T=750 \mathrm{~K}$ to $T=550 \mathrm{~K}\left(\Delta E_{b}=20 \mathrm{kcal} / \mathrm{mol}\right)$. 


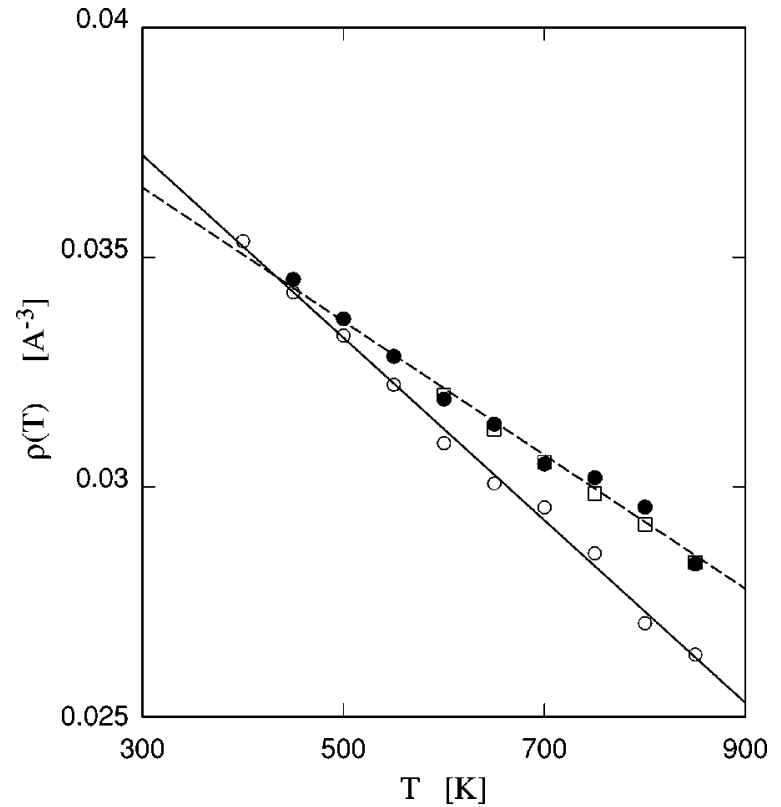

FIG. 13. Temperature dependence of the density for the polymer phase (solid dots, $\Delta E_{b}=18 \mathrm{kcal} / \mathrm{mol}$; open squares, $\Delta E_{b}=21 \mathrm{kcal} / \mathrm{mol}$ ) and for a molecular liquid comprising $\mathrm{S}_{8}$ rings (circles). The solid line is a linear interpolation of the data for the molecular liquid; the dashed line is a linear interpolation for the polymer phase with $\Delta E_{b}=18 \mathrm{kcal} / \mathrm{mol}$. The lines cross at $T=433 \mathrm{~K}$

inclusion leads to comparable mass fraction of rings in both cases. This suggests that the inclusion of bending and torsional force constants (neglected in polycarbonate) does not change significantly the relative weight of chains and rings. It selects a single size $\left(\mathrm{S}_{8}\right)$ as the dominant ring species, setting the stage for the reversible transformation to a $\mathrm{S}_{8}$ liquid below the $\lambda$ point. These observations support and complement the results of mean field models, ${ }^{26}$ predicting that bond bending and torsional forces do not affect significantly the relative abundance of monomers and polymers.

Polymerization reduces the thermal expansion coefficient, ${ }^{11}$ and this is illustrated in Fig. 13, where we show the $T$ dependence of the densities $\rho(T)$ of polymerized samples and a liquid comprising $\mathrm{S}_{8}$ rings. Both curves are approximately linear, and-assuming that polymerization is a continuous transition- the crossing of the two lines at $T_{f}$ $=433 \mathrm{~K}$ provides an estimate of the polymerization temperature for the model. The density of the polymer phase depends only weakly on $\Delta E_{b}$ (for $\Delta E_{b} \geqslant 18 \mathrm{kcal} / \mathrm{mol}$, see Fig. 13), but it is difficult to assess the errors in our estimate of $T_{f}$. An error bar of $\pm 10 \mathrm{~K}$ is obtained by considering statistical uncertainties in the evaluation of the average densities shown in Fig. 13.

Analysis of the chain conformations shows no unusual trends as a function of $T$. The mean-square distance between the two opposite chain heads grows linearly with the chain length over the entire polymer range, as expected for a Gaussian conformation. However, even in well-polymerized samples the length of the chains and their number are insufficient to allow a precise identification of small deviations from the linear dependence of square distance on chain length. The radial distribution function is little affected by

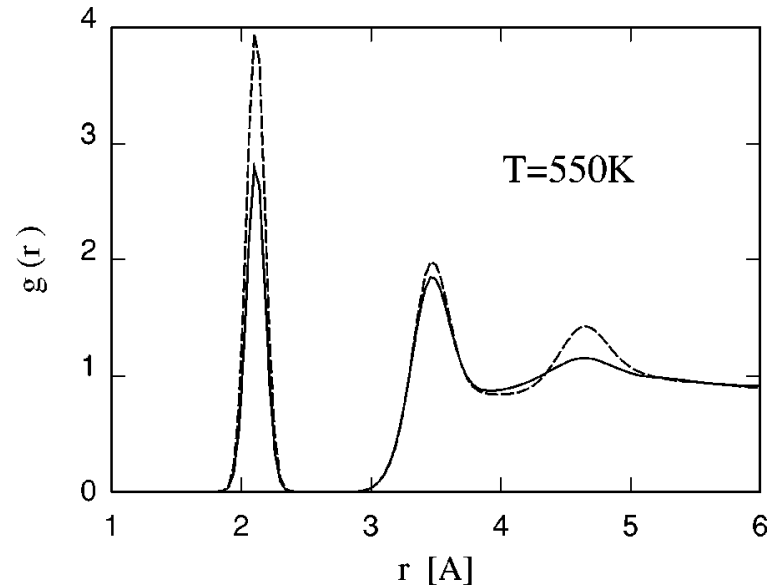

FIG. 14. Radial distribution function at $T=550 \mathrm{~K}$. Solid line: equilibrium polymer phase $\left(\Delta E_{b}=18 \mathrm{kcal} / \mathrm{mol}\right)$. Dashed line: $\mathrm{S}_{8}$ molecular phase.

polymerization, but the third peak changes substantially (see Fig. 14), as found experimentally. ${ }^{14,15}$ The computation of the constant-pressure specific heat shows no significant anomaly over the temperature range for which equilibration is achieved.

\section{POLYMERIZATION TRANSITION}

\section{A. Estimate of the transition temperature}

Although the present simulations cannot reach equilibrium at temperatures close to the expected polymerization point $(400 \leqslant T \leqslant 450)$, simulations at higher $T$ provide sufficient data for an extrapolation to this range. The simulation data for $\langle L\rangle(T)$ are reproduced fairly well (see Fig. 15 for $\Delta E_{b}=18 \mathrm{kcal} / \mathrm{mol}$ ) by the simple analytic function

$$
\langle L\rangle(T)=\left[A\left(T-T_{p}\right)+B\left(T-T_{p}\right)^{2}\right] \exp (-\alpha T)
$$

and $T_{p}$ can be considered an estimate of the polymerization temperature $T_{f}$. The fit results ( $T_{p}=430,452,455$, and 457 $\mathrm{K}$ for $\Delta E_{b}=15,18,20$, and $21 \mathrm{kcal} / \mathrm{mol}$ ) display only a weak dependence of $T_{p}$ on $\Delta E_{b}$ for $\Delta E_{b} \geqslant 18 \mathrm{kcal} / \mathrm{mol}$, thus

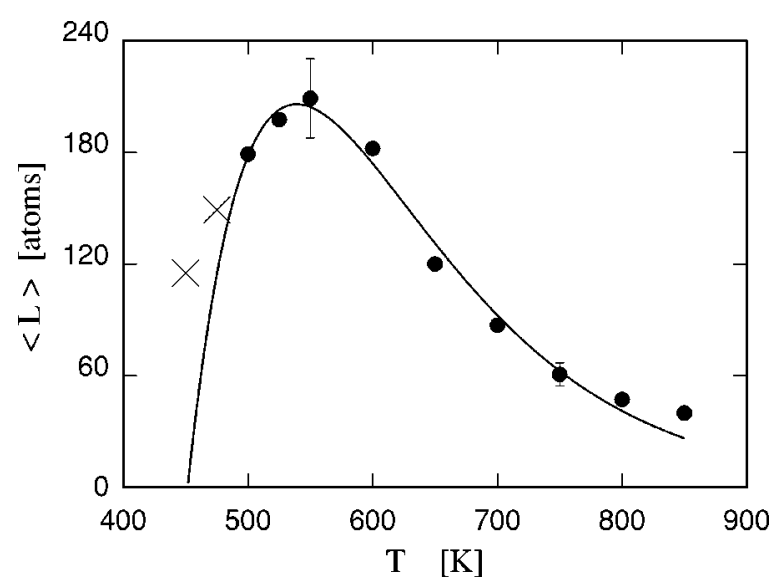

FIG. 15. Fit of the temperature dependence of $\langle L\rangle(T)$ for $\Delta E_{b}$ $=18 \mathrm{kcal} / \mathrm{mol}$ using the form of Eq. (7). The two crosses represent systems obtained by quenching from $T=550 \mathrm{~K}$ and give an upper bound for $\langle L\rangle(T)$ at temperatures for which equilibrium is not achieved by simulation. Representative error bars are shown. 
allowing us to identify $T_{p}=460 \mathrm{~K}$ as the polymerization temperature $T_{f}$ of the model in the limit of large $\Delta E_{b}$.

For each value of $\Delta E_{b}$, the linear term in the preexponential factor of Eq. (7) dominates, implying that the fit is continuous at $T_{p}$, but with a discontinuous first derivative. Being based on an extrapolation, this cannot be a firm assignment of the nature of the transition. However, the results for the density $\rho(T)$ of the molecular and polymer phases cross at $435 \mathrm{~K}$, and at $T=460 \mathrm{~K}$ they differ by less that $0.3 \%$. Any discontinuity of $\rho(T)$ at the transition is very small, and the first derivative $d \rho(T) / d T$ is discontinuous at $T_{f}$.

The saturation of $N_{24}(T)$ at $\sim 85 \%$ of the total mass over a wide temperature range makes it difficult to fit its $\Delta E_{b}$ and $T$ dependence with a simple analytic form. However, using the form of Eq. (7) and accepting a somewhat higher $\chi^{2}$, we obtained results equivalent to those provided by $\langle L\rangle(T)$, with an estimate of the transition temperature of $450 \mathrm{~K}$. The complexity of the fit and the extrapolation make it difficult to assign an error bar to these estimates. If the scatter of the three independent estimates of $T_{p}$ obtained by (a) fitting $\langle L\rangle(T)\left(T_{p}=460 \mathrm{~K}\right)$, (b) fitting $N_{24}(T)\left(T_{p}=450 \mathrm{~K}\right)$, and (c) plotting the densities of the molecular and of the polymer phase $\left(T_{p}=433 \mathrm{~K}\right)$ is a measure of the uncertainty in the determination of $T_{p}$, our estimate of the polymerization temperature is $T_{f}=450 \pm 20 \mathrm{~K}$.

\section{B. Polymerization transition and the average number of broken bonds}

The atoms in the $\alpha-\mathrm{S}_{8}$ molecular crystal define a dual lattice of bonds, which-in our model—may break at any $T>0 \mathrm{~K}$ because of thermal fluctuations. The average number $N_{b}$ of broken bonds is given by the Boltzmann expression

$$
N_{b}(T) / N_{\text {tot }}=\exp \left(-\Delta E_{b} / k_{B} T\right)
$$

where $N_{\text {tot }}$ is the total number of atoms in the sample. This assumes that the concentration of broken bonds is low enough that they behave as independent excitations of the ground-state bond configuration.

The number $N_{u}$ of unsaturated atoms is equal to $2 N_{b}$, and therefore its dependence on $\Delta E_{b}$ and $T$ is given by

$$
N_{u}(T) / N_{\text {tot }}=2 \exp \left(-\Delta E_{b} / k_{B} T\right) .
$$

If atoms can move and interchange bonds, however, the simple counting rules leading to Eqs. (8) and (9) are no longer valid, and it is more appropriate to consider unsaturated atoms as a dilute component whose potential energy per particle includes a $\Delta E_{b} / 2$ contribution due to the broken bonds. In this case, the concentration of unsaturated atoms is

$$
N_{u} / N_{\text {tot }}=\exp \left(-\Delta E_{b} / 2 k_{B} T\right) .
$$

Halving the exponent enhances significantly the population of unsaturated atoms beyond the low-temperature, static case. The fraction of unsaturated atoms in polymerized samples follows Eq. (10) closely (see Fig. 16), confirming the relevance of the dilute vapor picture for these atoms. This view is also supported by the far higher mobility of unsaturated atoms, since they are more weakly bound, and bond interchanges provide an additional mechanism for diffusion.

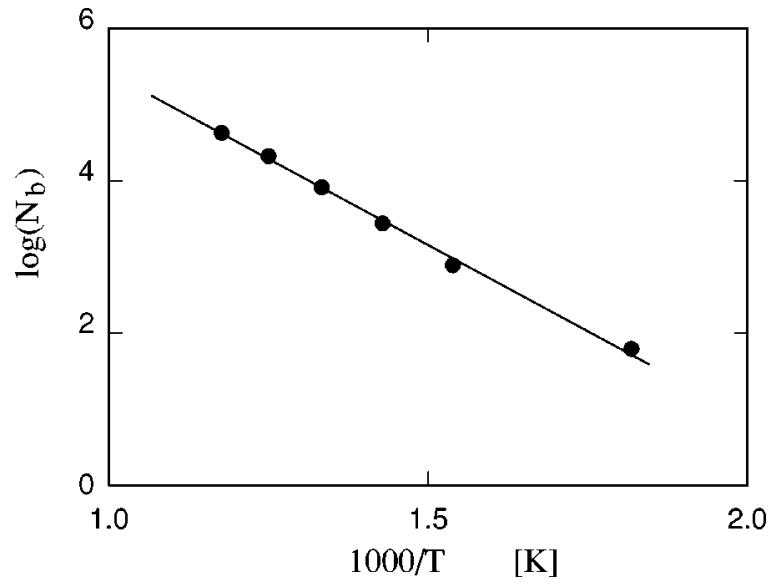

FIG. 16. Average number $N_{b}$ of broken bonds in polymeric samples as a function of inverse temperature. The solid line is a linear fit with angular coefficient equal to $-\Delta E_{b} / 2$. Error bars are comparable to the size of the symbols.

The density of unsaturated atoms in crystal samples is too low to verify the validity of Eq. (10) for temperatures where the crystal is stable and for the values of $\Delta E_{b}$ used here.

\section{DISCUSSION}

The equilibrium polymerization of liquid sulfur has been investigated by Monte Carlo simulations. The interatomic potential consists of a flexible intramolecular contribution and a Lennard-Jones intermolecular part, and the parameters have been determined by fitting the results of recent density functional computations for small sulfur rings and chains. ${ }^{32}$ The potential energy scheme goes beyond earlier work on sulfur by allowing the breaking and formation of covalent bonds. The parameter $\Delta E_{b}$ represents the energy cost of breaking a bond or energy gain upon forming a new one. Bond breaking and formation allow the system to approach equilibrium with respect to the bond configuration. The kinetics of bond equilibration is strongly enhanced by introducing a further relaxation mechanism, corresponding to bond interchanges around unsaturated chain terminations, following the pattern illustrated in Fig. 1.

The equilibrium concentration of unsaturated atoms in the liquid phase is proportional to the exponential of $\left[-\Delta E_{b} / 2 k_{B} T\right]$, which prevents the present simulations from reaching equilibrium for values of $\Delta E_{b}$ near the density functional $(25 \mathrm{kcal} / \mathrm{mol})$ or experimental $(32-36 \mathrm{kcal} / \mathrm{mol})$ estimates. Nevertheless, equilibrium is reached over a domain of $\Delta E_{b}$ and $T$ sufficiently wide to allow the investigation of the polymer phase and the dependence of the results on $\Delta E_{b}\left(T \geqslant 450 \mathrm{~K}\right.$ for $\Delta E_{b}=15 \mathrm{kcal} / \mathrm{mol}, T \geqslant 500 \mathrm{~K}$ for $\Delta E_{b}=18 \mathrm{kcal} / \mathrm{mol}, T \geqslant 550 \mathrm{~K}$ for $\Delta E_{b}=20$, and $T \geqslant 600 \mathrm{~K}$ for $\Delta E_{b}=21 \mathrm{kcal} / \mathrm{mol}$ ). Above these temperatures (the highest temperature of our simulations was $T=850 \mathrm{~K}$ ), liquid samples comprising $S_{8}$ molecules polymerize when the system is allowed to relax its bond configuration.

Polymerization is accompanied by an enthalpy increase $\Delta H$. Analysis of the $\Delta E_{b}$ dependence of $\Delta H$ allows us to estimate $\Delta H_{P}=3.85 \mathrm{kcal} / \mathrm{mol} \mathrm{S}_{8}$, in fair agreement with the 
experimental result $\Delta H_{P}=4.5 \mathrm{kcal} / \mathrm{mol} .{ }^{18}$ This result confirms that polymerization is entropy driven. Simple considerations show that translational entropy opposes polymerization, while vibrational entropy is nearly unchanged across the transition. By analogy to earlier model calculations, ${ }^{42,43}$ this suggests that polymerization is due to the entropy associated to the distribution of bonds among sulfur atoms. This picture confirms that the polymerization of sulfur is a thermodynamic transition, and not simply a progressive change in the chemical equilibrium of different $S_{n}$ species. ${ }^{14}$

The polymerized fraction displays a nonmonotonic dependence on $T$, with a maximum at $T_{\max }\left(\Delta E_{b}\right)$ exceeding $80 \%$ of the total system mass. $T_{\max }$ increases slightly with increasing $\Delta E_{b}$. A direct comparison with experimental results is difficult, because measurements are available only up to $T=560 \mathrm{~K}$, thus overlapping only slightly the simulations at the lowest $\Delta E_{b}$ values.

The polymer coexists in all samples with a significant population of $S_{8}$ molecules, whose relative weight increases rapidly with decreasing $T$. The molecular size distribution function for all other species follows a Zimm-Schulz distribution, whose parameters depend in a predictable way on $\Delta E_{b}$ and $T$. Molecules with more than 24 atoms are almost always open chains, and the concentration of rings other than $\mathrm{S}_{8}$ is low at all $T$. Comparison with polycarbonate results ${ }^{42,43}$ show that the bending and torsional contributions to the potential energy enhance the population of $S_{8}$ rings over all others, without changing significantly the relative weight of chains and rings. The validity of early renormalization group analyses of sulfur polymerization based on the $n \rightarrow 0$ model requires a vanishing concentration of rings. This assumption is not consistent with the present results, which predict a large concentration of $\mathrm{S}_{8}$ rings that increases with decreasing $T \rightarrow T_{f}$.

The thermal expansion coefficient of the polymer phase is lower than that of the liquid comprising $\mathrm{S}_{8}$ molecules only. The temperature dependence of the density in each is approximately linear over a wide temperature range, and the linear interpolations for the two data sets cross at $T=433$ $\pm 10 \mathrm{~K}$. This crossing point depends only very slightly on $\Delta E_{b}$. Assuming that the temperature dependence of the density is continuous across the transition and that only its slope $d \rho(T) / d T$ (directly related to the thermal expansion coefficient) changes discontinuously, this point $(T=433 \pm 10 \mathrm{~K})$ is our first estimate for the polymerization temperature of the model. Independent estimates are obtained by fitting the temperature dependence of the average chain length $\left(T_{f}\right.$ $=460 \mathrm{~K})$ and of the polymerized fraction $N_{24}\left(T_{f}\right.$ $=450 \mathrm{~K})$. From these three values, which are based on the assumption that the variable is continuous across the transition, with a discontinuous first derivative with respect to $T$, we predict a polymerization temperature $T_{f}=450 \pm 20 \mathrm{~K}$.

The good agreement between computed and measured $\Delta H_{P}$ and $T_{f}$ implies that the entropy change on polymerization $\left(\Delta S_{P}\right)$ is predicted reliably by the model. This quantity is not directly related to any property used to define the model, and its prediction suggests that $\Delta S_{P}$ mainly reflects the relative abundance of small rings and long chains, independent of the details of the interatomic potential. This could explain why selenium, which has a similar polymerization mechanism, displays an almost identical $\Delta S_{P} \cdot{ }^{45}$

A recent analysis of Raman data for liquid sulfur ${ }^{16}$ has provided a new quantitative determination of the polymerization fraction $\phi$ as a function of temperature for $408 \mathrm{~K} \leqslant T$ $\leqslant 573 \mathrm{~K}$. Comparison with the simulation results show that $N_{24}$ overestimates $\phi(T)$ somewhat, possibly due to the different definitions of $N_{24}$ and $\phi$, whose precise correspondence with molecular sizes is unclear. ${ }^{46}$ Our results predict that $\phi(T)$ has a maximum at a temperature $\sim 100 \mathrm{~K}$ above the temperature range explored in Ref. 16.

Our model is related to continuum versions of the lattice bond fluctuation model (BFM) ${ }^{47}$ which has been used extensively to investigate equilibrium polymers. ${ }^{48}$ Our work differs from most such studies because bond stretching, bending, and torsional parameters are included explicitly, the equilibrium of chains and rings is accounted for, and changes in the bond configuration are not limited to monomer addition and deletion at the chain terminations. Moreover, we use a potential incorporating realistic values for the interaction parameters, as our aim is to describe sulfur quantitatively. In this respect, our study is close in spirit to those of Refs. 36 and 49 , but describes better the energy of small sulfur rings and includes detailed information from new DF calculations. We emphasize the special role of covalent bonds by treating them as discrete binary variables (on-off), while in Ref. 36 they were defined in terms of interatomic distances. Of course, the vast improvement in computer facilities in recent years allows simulations far beyond those feasible in $1986 .{ }^{36}$

Our results suggest several directions for further work. First, it would be interesting to approach the polymerization point closer and to determine its character as a thermodynamic transition. Extending the present simulations to longer runs would help, but major improvements are required for a direct analysis of the transition point and its immediate surroundings. It would also be possible to raise the transition temperature to a range more amenable to computer simulation by diluting sulfur in an appropriate solvent, a strategy familiar from experiments. A final problem concerns the similarities and differences between the polymerization of sulfur and of selenium ${ }^{45}$ and their origin in the interatomic parameters and interactions.

\section{ACKNOWLEDGMENTS}

The calculations were performed in the Forschungszentrum Jülich on Athlon XP computers and Compaq XP1000 workstations provided in part by the Bundesministerium für Bildung und Wissenschaft, Bonn, within the MaTechKompetenzzentrum "Werkstoffmodellierung" (03N6015). We thank J. F. Douglas and S. C. Greer for helpful discussions, and R. Steudel for correspondence and information about the literature.

\footnotetext{
${ }^{1}$ S. C. Greer, Annu. Rev. Phys. Chem. 53, 173 (2002); J. Phys. Chem. B 102, 5413 (1998).

${ }^{2}$ J. Donohue, The Structure of the Elements (Wiley, New York, 1974), Chap. 9.

${ }^{3}$ B. Meyer, Chem. Rev. 76, 367 (1976).

${ }^{4}$ R. Steudel, in Studies in Inorganic Chemistry, edited by A. Müller and B. Krebs (Elsevier, Amsterdam, 1984), Vol. 5, p. 3, and references therein.
} 
${ }^{5}$ The melting point of the monoclinic $\beta$ phase at $P=1 \mathrm{~atm}$ is $T_{m}=386 \mathrm{~K}$. The liquid may form from other phases at slightly different temperatures. ${ }^{6}$ R. Steudel, R. Strauss, and L. Koch, Angew. Chem. 97, 58 (1985).

${ }^{7}$ A recent review of liquid sulfur is given by R. Steudel, Top. Curr. Chem. (to be published).

${ }^{8}$ The color change from yellow to red might not be observable for highly purified samples. See K. M. Zheng and S. C. Greer, J. Chem. Phys. 96, 2175 (1992).

${ }^{9}$ E. D. West, J. Am. Chem. Soc. 81, 29 (1959).

${ }^{10}$ F. Feher, G. P. Goerler, and H. D. Lutz, Z. Anorg. Allg. Chem. 382, 135 (1971).

${ }^{11}$ K. M. Zheng and S. C. Greer, J. Chem. Phys. 96, 2175 (1992).

${ }^{12}$ R. F. Bacon and R. Fanelli, J. Am. Chem. Soc. 65, 639 (1943).

${ }^{13}$ J. Ruiz-Garcia, E. M. Anderson, and S. C. Greer, J. Phys. Chem. 93, 6980 (1989).

${ }^{14}$ (a) R. Winter, C. Szornel, W. C. Pilgrim, W. S. Howells, P. A. Egelstaff, and T. Bodensteiner, J. Phys.: Condens. Matter 2, 8427 (1990); (b) M. Stolz, R. Winter, W. S. Howells, R. L. McGreevy, and P. A. Egelstaff, ibid. 6, 3619 (1994).

${ }^{15}$ R. Bellissent, L. Descotes, F. Boué, and P. Pfeuty, Phys. Rev. B 41, 2135 (1990).

${ }^{16}$ A. G. Kalampounias, K. S. Andrikopoulos, and S. N. Yannopoulos, J. Chem. Phys. 118, 8460 (2003).

${ }^{17}$ M. Schmidt, Angew. Chem. 85, 474 (1973).

${ }^{18}$ R. Steudel, Phosphorus Sulfur 16, 251 (1983); R. Steudel, S. PasslackStephan, and G. Holdt, Z. Anorg. Allg. Chem. 517, 7 (1984).

${ }^{19}$ M. A. Anisimov, K. I. Kugel, and T. Y. Lisovskaya, High Temp. 25, 165 (1987).

${ }^{20}$ G. Gee, Trans. Faraday Soc. 48, 515 (1952).

${ }^{21}$ A. V. Tobolsky and A. Eisenberg, J. Am. Chem. Soc. 81, 780 (1959).

${ }^{22}$ R. L. Scott, J. Phys. Chem. 69, 261 (1965).

${ }^{23}$ R. E. Powell and H. Eyring, J. Am. Chem. Soc. 65, 648 (1943).

${ }^{24}$ J. C. Wheeler, S. J. Kennedy, and P. Pfeuty, Phys. Rev. Lett. 45, 1748 (1980).

${ }^{25}$ S. J. Kennedy and J. C. Wheeler, J. Chem. Phys. 78, 1523 (1983).

${ }^{26}$ (a) J. Dudowicz, K. F. Freed, and J. F. Douglas, J. Chem. Phys. 111, 7116 (1999); (b) 112, 1002 (2000); (c) 113, 434 (2000).

${ }^{27}$ V. F. Kozhevnikov, J. M. Viner, and P. C. Taylor, Phys. Rev. B 64, 214109 (2001).

${ }^{28}$ T. P. Martin, J. Chem. Phys. 81, 4426 (1984); S. Hunsicker, R. O. Jones, and G. Ganteför, ibid. 102, 5917 (1995), and references therein.

${ }^{29}$ M. W. Wong, Y. Steudel, and R. Steudel, Chem. Phys. Lett. 364, 387
(2002), and references therein; L. Peter, Phosphorus, Sulfur Silicon Relat. Elem. 168, 287 (2001) and (private communication); S. Millefiori and A. Alparone, J. Phys. Chem. A 105, 9489 (2001).

${ }^{30}$ M. Springborg and R. O. Jones, J. Chem. Phys. 88, 2652 (1988).

${ }^{31}$ D. Hohl, R. O. Jones, R. Car, and M. Parrinello, J. Chem. Phys. 89, 6823 (1988).

${ }^{32}$ R. O. Jones and P. Ballone, J. Chem. Phys. 118, 9257 (2003).

${ }^{33}$ J. S. Tse and D. D. Klug, Phys. Rev. B 59, 34 (1999).

${ }^{34}$ R. Car and M. Parrinello, Phys. Rev. Lett. 55, 2471 (1985).

${ }^{35}$ D. J. Brunelle, in Ring-Opening Polymerization: Mechanisms, Catalysis, Structure, Utility, edited by D. J. Brunelle (Hanser, München, 1993), p. 309.

${ }^{36}$ F. H. Stillinger, T. A. Weber, and R. A. LaViolette, J. Chem. Phys. 85, 6460 (1986).

${ }^{37}$ C. Pastorino and Z. Gamba, J. Chem. Phys. 112, 282 (2000); Chem. Phys. 261, 317 (2000); Chem. Phys. Lett. 319, 20 (2000); E. Venuti, G. Cardini, and E. Castellucci, Chem. Phys. 165, 313 (1992).

${ }^{38}$ CRC Handbook of Chemistry and Physics, 81 st ed., edited by D. R. Lide (CRC, Boca Raton, FL, 2000).

${ }^{39}$ M. Parrinello and A. Rahman, J. Appl. Phys. 52, 7182 (1981).

${ }^{40}$ H. C. Andersen, J. Chem. Phys. 72, 2384 (1980).

${ }^{41}$ The choice of $L=24$ to separate the "molecular" and "polymer" ranges ensures that the latter are significantly different from $\mathrm{S}_{8}$, which is always the most abundant molecular species. Much larger cutoff values would not be appropriate for the sample size (2048 atoms).

${ }^{42}$ P. Ballone and R. O. Jones, J. Chem. Phys. 115, 3895 (2001).

${ }^{43}$ P. Ballone and R. O. Jones, J. Chem. Phys. 116, 7724 (2002).

${ }^{44}$ For example, the mass concentrations $(\%)$ in the sample equilibrated at $T=550 \mathrm{~K}$ with $\Delta E_{b}=18 \mathrm{kcal} / \mathrm{mol}$ were $\mathrm{S}_{7}: 0.46, \mathrm{~S}_{8}: 16.47, \mathrm{~S}_{9}: 0.088$, $\mathrm{S}_{12}: 0.059$.

${ }^{45}$ A. Eisenberg and A. V. Tobolsky, J. Polym. Sci. 46, 19 (1960).

${ }^{46}$ The uncertainty in the definition of $\phi(T)$ is confirmed by the difference ( $\sim 50 \%$ at $T=473 \mathrm{~K}$ ) between the estimates of Refs. 16 and 27, based on sound attenuation measurements.

${ }^{47}$ I. Carmesin and K. Kremer, Macromolecules 21, 2819 (1988); W. Paul, K. Binder, D. Heermann, and K. Kremer, J. Chem. Phys. 95, 7726 (1991).

${ }^{48}$ See, for instance, Y. Rouault and A. Milchev, Phys. Rev. E 51, 5905 (1995) and references therein.

${ }^{49}$ A simulation study based on a tight binding Hamiltonian for sulfur is reported in L. Descotes and C. Bichara, J. Non-Cryst. Solids 192-193, 627 (1995). 